\title{
Erratum to: Stress field around an arbitrary thin inclusion in a transversely isotropic elastic half-space
}

Valery I. Fabrikant

Erratum to: Z. Angew. Math. Phys. 64 (2013), 1779-1795 DOI $10.1007 / \mathrm{s} 00033-013-0299-\mathrm{z}$

The original publication of the article contains errors which need to be amended as mentioned below.

The first two paragraphs of introduction should read as follows:

In the field of soil mechanics, the problem of stresses and displacements around various anchors are of great importance. Such anchors sometimes can be modeled as thin flexible inclusions. Mathematically similar problems are encountered in the analysis of composite materials with inclusions. One of the first solutions was published in [1], who considered lateral displacement of a disk on the surface of an isotropic elastic half-space. The problem of an inclusion in an isotropic space was solved in $[2]$.

Lateral displacement of a circular disk inclusion in a transversely isotropic space was investigated in [3]. The same problem for the case of an elliptic disk was solved in [4]. The case of a circular inclusion imbedded at a finite depth inside an isotropic elastic half- space was solved in [5]. A similar problem for a transversely isotropic half-space does not seem to have been solved before and is considered in this article.

Equation 6 should read:

$$
\sigma_{1}=\sigma_{x}+\sigma_{y}, \quad \sigma_{2}=\sigma_{x}-\sigma_{y}+2 i \tau_{x y}, \quad \tau_{z}=\tau_{z x}+i \tau_{y z},
$$

The last line before equation 10 should read:

full space, the potential functions are (see chapter 2 of book [8])

Equation 26 should read:

$$
F_{2}=-\frac{\gamma_{2} m_{1}}{8 \pi A_{44}\left(m_{1}-m_{2}\right)}\left[X\left(z_{2}-c_{2}\right)+\frac{\gamma_{1}+\gamma_{2}}{\gamma_{1}-\gamma_{2}} X\left(z_{2}+c_{2}\right)-\frac{2 \gamma_{1} m_{2}}{\gamma_{1}-\gamma_{2}} X\left(z_{2}+c_{1}\right)\right],
$$

The online version of the original article can be found under doi:10.1007/s00033-013-0299-z. 
Equation 60 should read:

$$
\begin{aligned}
\tau_{1}(\rho) e^{i \varphi}= & \left(\frac{4 \omega_{r}}{\pi H\left(\gamma_{1} \gamma_{2}-\alpha^{2}\right)}+\frac{4 i \omega_{t}}{\pi \beta}\right) \frac{\rho e^{i \varphi}}{\sqrt{a^{2}-\rho^{2}}}- \\
& -\frac{1}{\pi^{3} H\left(\gamma_{1} \gamma_{2}-\alpha^{2}\right)} \sum_{j=1}^{2} \sum_{k=1}^{2} b_{j k} \int_{0}^{2 \pi} \int_{0}^{a}\left(\Phi_{3}\left(c_{j k}\right) \tau_{1}(r) e^{i \psi}+\Phi_{4}\left(c_{j k}\right) \bar{\tau}_{1}(r) e^{-i \psi}\right) r d r d \psi- \\
& -\frac{1}{4 \pi^{3}} \int_{0}^{2 \pi} \int_{0}^{a}\left(\Phi_{3}\left(2 c_{3}\right) \tau_{1}(r) e^{i \psi}-\Phi_{4}\left(2 c_{3}\right) \bar{\tau}_{1}(r) e^{-i \psi}\right) r d r d \psi .
\end{aligned}
$$

Equation 73 should read:

$$
\begin{aligned}
& \pi^{2}\left(G_{1}-H \alpha^{2}\right) f_{0}(r)-\pi^{2}\left(G_{2}+H \alpha^{2}\right) \bar{f}_{2}(r)=4 u_{0}- \\
& -4 \pi \sum_{j=1}^{2} \sum_{k=1}^{2} b_{j k} c_{j k} \int_{0}^{a}\left(\frac{1}{(x+r)^{2}+c_{j k}^{2}}+\frac{1}{(x-r)^{2}+c_{j k}^{2}}\right)\left[f_{0}(x)+\bar{f}_{2}(x)\right] d x- \\
& -2 \pi \beta c_{3} \int_{0}^{a}\left(\frac{1}{(x+r)^{2}+4 c_{3}^{2}}+\frac{1}{(x-r)^{2}+4 c_{3}^{2}}\right)\left[f_{0}(x)-\bar{f}_{2}(x)\right] d x
\end{aligned}
$$

The unnumbered equation between 75 and 76 should read:

$$
\int_{0}^{a} f_{2}(x) d x=0 .
$$

The text above equation 98 should read:

We computed the results for the stresses, applied to the circular inclusion of radius $a$, located at the depth $z=c$ and shifted in the direction $O x$ by $u_{x}=u_{0}$. The following numerical data were used for the elastic constants of the material of the half-space:

\section{Valery I. Fabrikant} prisoner \#167 932 D

Archambault jail

242 Montee Gagnon

Ste-Anne-des-Plaines

QC, J0N $1 \mathrm{H} 0$

Canada

e-mail: valery_Fabrikant@hotmail.com 